\title{
X-linked Cornelia de Lange Syndrome - Remembering a dysmorphology case in Neonatology
}

\author{
Ana Rita Soares ${ }^{1 *}$, Gonçalo Inocêncio² ${ }^{2}$ Céu Rodrigues², Elisa Proença ${ }^{3}$, Gabriela Soares ${ }^{1}$, Ana Maria Fortuna',
} Céu Mota ${ }^{3}$

'Medical Genetics Department, Centro de Genética Médica Doutor Jacinto Magalhães, Porto, Portugal ${ }^{2}$ Obstetrics/Gynecology Department, Centro Materno-Infantil do Norte, Porto, Portugal

${ }^{3}$ Neonatology Department, Centro Materno-Infantil do Norte, Porto, Portugal

Article Info

\section{Article Notes}

Received: December 14, 2017

Accepted: January 04, 2018

\section{*Correspondence:}

Dr. Ana Rita Soares, Medical Genetics Department, Centro de Genética Médica Doutor Jacinto Magalhães, Porto, Portugal; E-Mail: anaritamsoares@gmail.com

C) 2018 Soares RA. This article is distributed under the terms of the Creative Commons Attribution 4.0 International License.

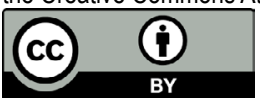

Keywords:

Cornelia de Lange Syndrome

Dysmorphology

Neonatology

Perinatal period

Growth restriction

SMC1A gene

\section{ABSTRACT}

Cornelia de Lange Syndrome is a rare disease characterized by prenatal and postnatal growth restriction, mild to severe intellectual disability, characteristic facial dysmorphisms, and other anomalies. The diagnosis is based on clinical criteria or on detection of a pathological variant in one of the five disease causing genes - NIPBL, RAD21, SMC3, HDAC8 or SMC1A. The prognosis may depend on the precocity of malformations' treatment, early institution of therapies and careful follow-up of possible complications. We present a case of Cornelia de Lange Syndrome whose clinical diagnosis was made during perinatal period due to a suspicious prenatal history followed by characteristic dysmorphisms and anomalies. The molecular confirmation was only possible after two years, revealing a pathogenic variant in SMC1A gene, located at X chromosome, which occurred as de novo event. The clinical suspicion during perinatal period made it possible to start an earlier multidisciplinary follow-up and to offer a better management for the child and a proper genetic counseling for the parents.

\section{Introduction}

Cornelia de Lange Syndrome is a rare disease characterized by facial dysmorphisms (which are the hallmark of the syndrome and include synophrys and arched eyebrows, long thick eyelashes, small nose with anteverted nares and flat bridge, down-slanting thin lips, sparse teeth, high palate, long soft philtrum, micrognathia, posterior rotated low-set ears and short neck), prenatal and postnatal growth restriction, low weight and microcephaly, mild to severe intellectual disability, behavior alterations, forearms reduction defects and other musculoskeletal anomalies, neurosensorial or skin anomalies and others, including congenital cardiac disease, gastrointestinal and genitourinary anomalies ${ }^{1}$. In prenatal set, ultrasound findings may include increased nuchal translucency, growth failure, the typical in utero facial profile and limb malformations, although they may not be present and are not specific. The diagnosis is based on clinical criteria or on detection of a pathological variant in one of the five disease causing genes - NIPBL, RAD21, or SMC3 (with autosomal dominant inheritance) and HDAC8 or SMC1A (with $\mathrm{X}$-linked inheritance). Molecular confirmation is possible in about $70 \%$ of cases and the syndrome may be inherited or occur as de novo event. The most common one is NIPBL gene ( $60 \%$ of positive cases). SMC1A gene is found in $5 \%$ of cases ${ }^{2,3}$. There is no cure available for 
this syndrome and management guidelines were proposed by Kline group ${ }^{1}$. They include: plotting growth parameters on CdLS-specific growth charts, gastrointestinal and nutritional evaluations, radiographs of the upper extremities, audiology and ophthalmology evaluations, echocardiogram, neurologic evaluation and EEG, renal ultrasonography and vesicoureterogram (if necessary), urologic evaluation in males and pelvic ultrasound in females, complete blood count, multidisciplinary developmental evaluation and consultation with clinical geneticist. The prognosis may depend on the precocity of malformations' treatment, early institution of therapies and careful follow-up of possible complications ${ }^{1,3}$.

\section{Case Report}

We present a newborn girl, second child of healthy nonconsanguineos parents, with no relevant family history.

During pregnancy, due to positive 1 st trimester screening (PAPP-A and free $\beta$-HCG from maternal blood associated with nuchal translucency and nasal bones in ultrasound and maternal age), an amniocentesis was performed and revealed a normal female karyotype. Maternal serologies were normal. Prenatal ultrasound revealed single umbilical artery and placenta previa. At 29 gestational weeks, the diagnosis of fetal growth restriction was made.

The girl was born after a c-section with weight, length and head circumference in 5-10 percentile (Fenton curves) and Apgar 4/8/9 at 1/5/10 minutes, needing mechanical ventilation via endotracheal tube.

She presented with facial dysmorphisms, hypertrichosis (Figures 1 and 2) and systolic murmur grade II/IV.

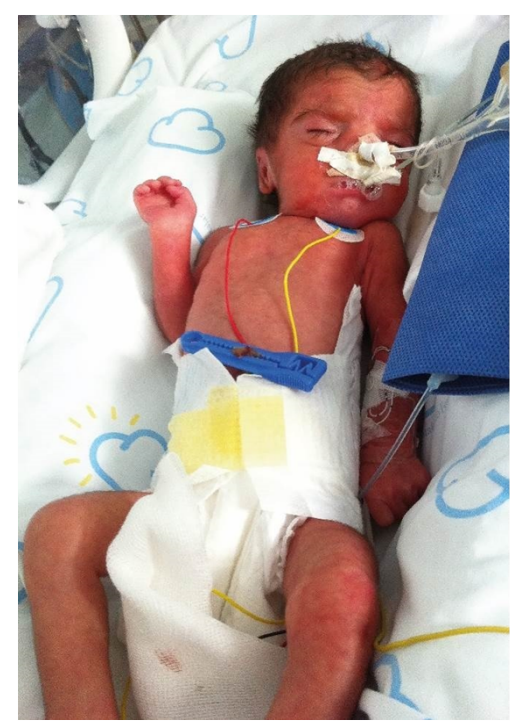

Figure 1. Newborn girl in Newborn Care Unit presenting with facial dysmorphisms (peculiar face with anteverted nares, ptosis and long eyelashes, low-set ears and low-set hair implantation) and hypertrichosis.

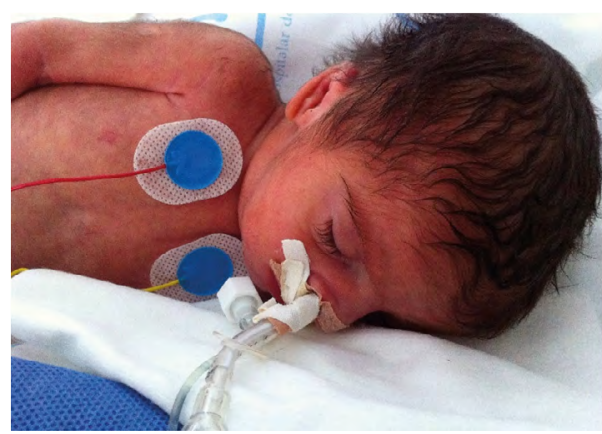

Figure 2. Newborn girl in Newborn Care Unit presenting with facial dysmorphisms - side view.

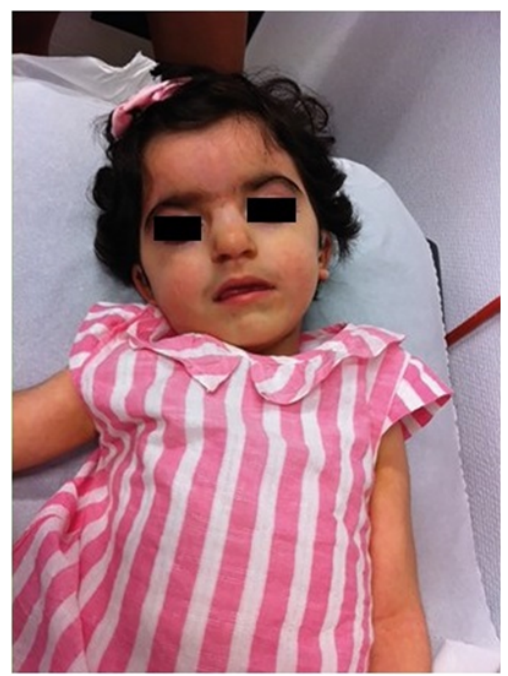

Figure 3. 2-year-old girl presenting with microcephaly and facial dysmorphisms (synophrys and arched eyebrows, ptosis and long eyelashes, anteverted nares, low-set ears and low-set hair implantation), hypertonia, cervical hyperextension and hearing aids.

Neurologic examination revealed axial hypotonia with joint stiffness and hip abduction limitation. Placental anatomic and cytogenetic studies were normal. She was admitted to Neonatal Intensive Care Unit, where several studies were performed and revealed peripheral pulmonary stenosis and patent foramen oval, severe gastroesophageal reflux and neurosensory deafness.

Due to facial dysmorphisms and clinical presentation, the diagnosis of Cornelia de Lange Syndrome was made. Molecular studies for NIPBL (both sequencing and MLPA studies) and SMC3 genes (sequencing) were performed, with negative results.

Nowadays, she is 2 years-old and she presents with the same facial dysmorphisms (Figure 3), synophrys, weight and high below 5 percentile, microcephaly and global developmental delay. She also presents with stereotypic movements of the hands, hypertonia, cervical hyperextension and increased osteotendinous reflexes, as well as hearing aids and glasses for myopia and 
strabismus (picture not shown). She also has swallowing difficulties, gastroesophageal reflux (medicated with domperidone), constipation, convulsive crises (medicated with levetiracetam $35 \mathrm{mg} / \mathrm{Kg} /$ day) and incomplete right bundle branch block.

Very recently, the molecular diagnosis was confirmed by Sanger sequencing with detection of the probably pathogenic variant c.1457A $>\mathrm{G}$ (p.Asp486Gly) in heterozygosity in the $S M C 1 A$ gene, located at $\mathrm{X}$ chromosome (NM_006306.3:c.1279G $>$ A: missense variant).

Parental studies revealed no alteration in SMC1A gene, so the recurrence risk given to the family was low, although higher than the general population (due to the possibility of gonadal mosaicism). Prenatal diagnosis or preimplantation genetic diagnosis was offered to the family in future pregnancies.

\section{Conclusion}

With this case, the authors wish to stress the importance of a multidisciplinary team, mainly in these rare and demanding diseases. Furthermore, this case is important to remember that a suspicious prenatal history followed by characteristic dysmorphisms presented during perinatal period can be a clue to do a diagnosis and start proper management earlier, giving the best care possible.

\section{Conflict of Interest}

The authors have no conflicts of interest to disclose.

\section{References}

1. Kline A, Krantz ID, Sommer A, et al. "Cornelia de Lange Syndrome: Clinical Review, Diagnostic and Scoring Systems, and Anticipatory Guidance". AJMG Part A. 2007; 143A: 1287-1296

2. Oliveira J, Dias C, Redeker E, et al. "Development of NIPBL locusspecific database using LOVD: from novel mutations to further genotype-phenotype correlations in Cornelia de Lange Syndrome", Hum Mutat. 2010 Nov; 31(11): 1216-22

3. Deardorff MA, Noon SE, Krantz ID, et al. Genereviews®: Cornelia de Lange Syndrome. Jan 2016. https://www.ncbi.nlm.nih.gov/books/ NBK1104/ 